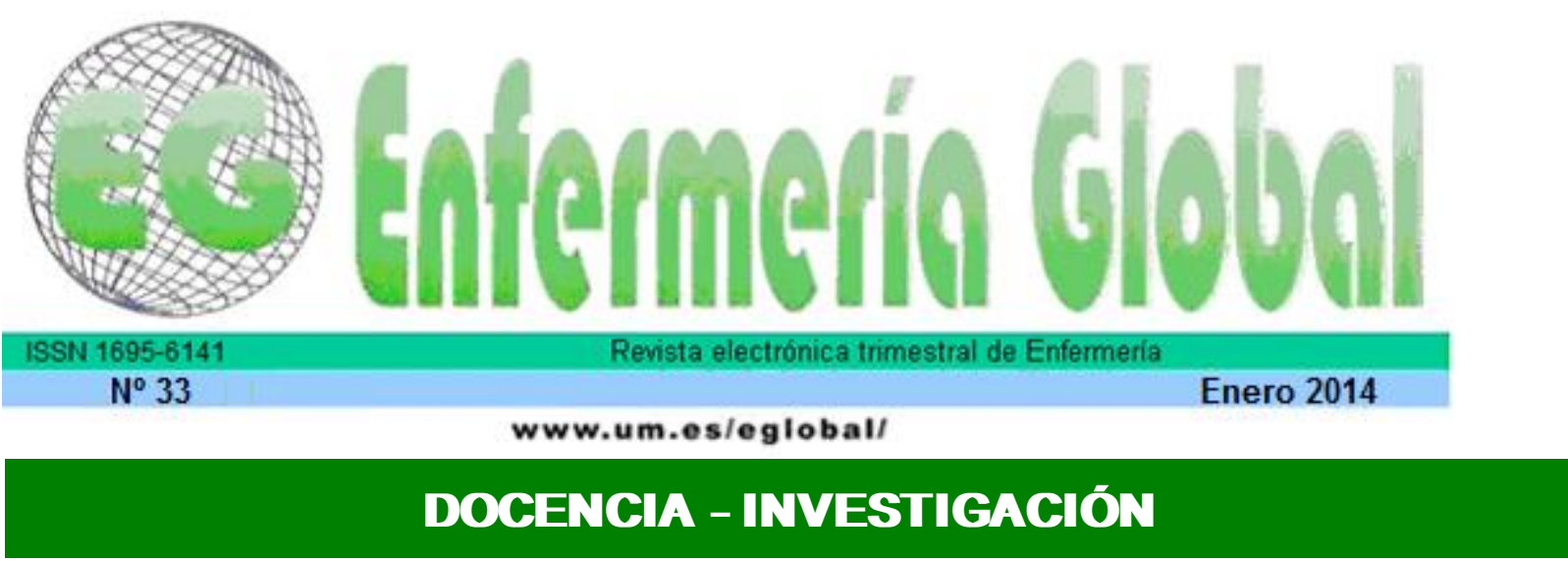

\title{
Reflexiones de los alumnos de Enfermería sobre el proceso de la muerte
}

Nursing Students' Reflections on the Death Process

\section{*Hanzeliková Pogrányivá, A ${ }^{* *}$ García López, MV ${ }^{* * *}$ Conty Serrano, MR ****ópez Davila Sánchez, B *****Barriga Martín JM ******Martín Conty JL}

\begin{abstract}
*Doctoranda en Calidad de cuidados por la Universidad Camilo José Cela de Madrid; Enfermera, Unidad de agudos - Hospital Virgen del Valle. Toledo. E-mail: alica.hanzelik@gmail.com **DUE.

Licenciada en Antropología. Profesora Titular Escuela Universitaria de Enfermería de Castilla La Mancha. Toledo *** DUE. Licenciada en Antropología. Doctora en Ciencias de la Educación. Profesora Titular Escuela Universitaria de Enfermería de Castilla La Mancha. Toledo **** Enfermera. Complejo Hospitalario. Toledo *****Doctorando en Psicología por la Universidad Camilo José Cela de Madrid; Enfermero Apoyo en Gestión. Hospital 12 de Octubre. Madrid ${ }^{* * * * * * D U E . ~ M a ́ s t e r ~ e n ~ R i e s g o s ~}$ Laborales; Profesor asociado Universidad Camilo José Cela de Madrid.
\end{abstract}

Palabras clave: muerte; estudiantes de enfermería; afrontamiento; paciente.

Keywords: death; nursing students; coping; patient.

\section{RESUMEN}

La actitud y el miedo a la muerte en enfermería pueden condicionar la calidad de cuidados del paciente moribundo, por lo que es importante abordar este tema.

Es necesario aprender a percibir la muerte como algo natural. Los sanitarios tratan a los moribundos, interpretan el fenómeno de la muerte y la muerte misma bajo su óptica y entendimiento. La formación en tanatología y los cuidados paliativos se trata de forma escasa o al menos de forma irregular en los planes de estudio.

El objetivo del presente trabajo es analizar las aportaciones de los alumnos de tercero de enfermería en la asignatura cuidados paliativos a la hora de escribir una reflexión sobre un artículo que trata la visión de una enfermera del proceso de la muerte.

Se empleó la fenomenología como instrumento metodológico, con el que se pretendió elaborar inductivamente un marco explicativo del fenómeno estudiado a partir de las expresiones de los estudiantes.

En relación a la actitud hacia el trabajo con enfermos terminales y hacia la muerte se pueden resaltar 
los siguientes datos: escasa preparación, afrontamiento ineficaz, miedos, valores y creencias. Predomina la inquietud relacionada con el encarnizamiento terapéutico y con la actitud obstinada del equipo de salud por salvar lo insalvable.

Los resultados ponen de manifiesto un grado muy elevado de implicación emocional de la enfermera en el cuidado del paciente moribundo. Se considera como pilar fundamental una preparación específica para adaptarse mejor al trabajo con los pacientes terminales. Destaca el interés y la preocupación por la excelencia.

Es muy importante el analizar los problemas en "entorno de la muerte" en los estudiantes de enfermería. La investigación cualitativa en este campo podría ser una de las herramientas útiles para detectar inquietudes y preocupaciones.

\section{ABSTRACT}

One's attitude toward and fear of death in the nursing field can determine the quality of care that a dying patient receives, which makes this topic an important one to consider and discuss.

It is necessary for one to learn to perceive death as a natural process. Healthcare workers treat dying patients, interpreting the phenomenon of death as well as death itself through their own personal lens and breadth of understanding. Training in thanatology and palliative care is scarcely and irregularly included in regular academic curricula.

The objective of this study is to analyze the contributions of $3^{\text {rd }}$-year nursing students on the subject of palliative care upon writing a reflective journal entry about an article that surrounds a nurse's view on the process of dying.

Phenomenology was used as a methodological instrument which intended to inductively draw up a standardized explanation of the phenomenon of death through the students' expressed thoughts on the matter.

Relating to the attitudes that nurses that work with the terminally ill have towards death, the following facts stand out as impactful: lack of preparation, ineffective coping, and personal fears, values, and beliefs. Additionally, uneasiness associated with the use of life support and the care team's obstinate insistence on saving what cannot be saved is predominant.

The results emphasize a very elevated level of the nurse's emotional involvement in caring for a dying patient. Specific preparation geared toward a better adaptation in working with terminal patients has been identified as a fundamental pillar for nurses in this line of work. Personal interest and emphasis on occupational excellence also stand out as influential factors.

It is very important to analyze the problems regarding this "environment of death" and its impact on nursing students. Qualitative research in this field could be a useful tool in detecting worries and uncertainties surrounding this matter.

\section{INTRODUCCIÓN}

Aunque la muerte es el fin inevitable de la vida humana, en la sociedad actual se habla muy poco de ella. Es necesario aprender a percibir la muerte como algo natural; parte de nuestra vida cotidiana. Los sanitarios tratan a los moribundos, interpretan el fenómeno de la muerte y la muerte misma bajo su óptica y entendimiento. La actitud y el miedo a la muerte en enfermería pueden condicionar la calidad de cuidados del paciente moribundo, por lo que es importante abordar esta formación. La formación en cuidados paliativos y la educación en tanatología y los cuidados paliativos están presentes de manera irregular en los actuales planes de estudios o es escasa.

Prácticamente todas las enfermeras en algún momento de su vida profesional se 
reúnen con la muerte. Tantos a los pacientes moribundos/terminales como a sus familiares deben proporcionarles un cuidado sensible. El trato que recibe el moribundo de los profesionales sanitarios está condicionado por cómo entienden los fenómenos de la muerte y el proceso de la muerte ${ }^{(1)}$ La falta o escasa preparación puede llevar a producir ciertas alteraciones en el nivel emocional en los profesionales. El trabajo en unidades de paliativos es altamente estresante ${ }^{(2-4)}$. La ansiedad, el miedo y el estrés pueden aparecer si no se desarrollan estrategias eficaces de afrontamiento.

La ansiedad se caracteriza generalmente como algo muy desagradable en el nivel del estado emocional. El individuo tiende a tener problemas, experimenta sentimientos de amenaza pero a menudo no es capaz de identificar la causa de su miedo y no puedo predecir cuándo y de dónde viene el peligro. La ansiedad está provocada por el temor a algo desconocido (el miedo "de nada en concreto") ${ }^{(5)}$.

El miedo se define como una desagradable sensación a corto plazo, estado emocional durante el curso, o sólo el estímulo esperado de los procesos cognitivos de la persona como algo amenazante o peligroso para ella. El individuo es, en general, capaz de identificar la causa de su miedo.

La ansiedad es por lo general orientada a la emoción, el futuro se caracteriza por la incertidumbre, las amenazas. El miedo es una respuesta inmediata, alarma de reacción a la amenaza actual ${ }^{(5)}$.

Algunos autores definen el miedo a la muerte ${ }^{(6)}$ como una experiencia humana en la vida cotidiana en una situación aguda en que está en peligro inminente de la vida. Incluye varios componentes: la preparación para su propia muerte, el proceso de morir y el miedo a la muerte de otros.

La ansiedad y el miedo a la muerte es algo normal. Se inculcan a menudo durante la socialización. Por lo tanto, el clima social desfavorable hacia el concepto de muerte y el morir y aspectos culturales pueden afectar a la experiencia del miedo y la ansiedad de la muerte. Existen también otros factores ${ }^{(4,7)}$ que influyen en el grado de ansiedad y miedo a la muerte: Salud mental y experiencias de la vida con la muerte. También puede influir la religión, la cultura, opinión sobre la calidad de vida y la muerte.

Educación y formación dirigida a la muerte y el morir para los estudiantes universitarios en disciplinas de la salud para reducir el miedo y la ansiedad de la muerte, por lo tanto, en la enseñanza de los cuidados paliativos para las enfermeras en general se debe incorporar el análisis de los problemas de la muerte y el miedo a ella ${ }^{(10)}$.

\section{OBJETIVO}

Analizar las aportaciones de los alumnos de tercero de enfermería en la asignatura cuidados paliativos del curso 2009/2010 de la Universidad Alfonso X a la hora de escribir una reflexión sobre el artículo de Gil PR., Ávila R, IM.; Molero PMJ. "Una visión enfermera del proceso de la muerte". ${ }^{(8)}$

\section{METODOLOGÍA}

Estudio cualitativo. Se empleó la fenomenología como instrumento metodológico, con el que se pretende elaborar inductivamente un marco explicativo del fenómeno 
estudiado a partir de las expresiones de los Estudiantes ${ }^{(11)}$. Los datos se han obtenido mediante 41 trabajos de reflexión sobre la lectura del artículo: Gil P. R,; Ávila R.; I.M.; Molero P.M.J.; "Una visión enfermera del proceso de la muerte" (8) En este articulo se presenta el relato de una enfermera, con 21 años de experiencia profesional en servicios de oncología, en el que explica sus vivencias, la evolución de sus sentimientos, así como su trayectoria.

Se entregaron a través del portal de la asignatura 4 artículos relacionados con la muerte, moribundos, enfermos terminales. Los alumnos pudieron elegir el texto que más les gustase de los textos propuestos, para realizar una reflexión.

El articulo "Una visión enfermera del proceso de la muerte" lo eligió el 38,5\% (N 41) de los alumnos, frente al 19,44\%, 8,25\%, 34,25\%. Sexo: 81,0\% mujeres; $19 \%$ hombres. La edad media $24,54 \%$ años.

Los datos se obtuvieron mediante la escritura reflexiva. La escritura reflexiva es un proceso cognitivo complejo mediante el cual la persona traduce sus ideas, pensamientos, sentimientos e impresiones en un discurso escrito coherente. El proceso favorece la comunicación compresiva con la audiencia y el logro de pretendidos objetivos $^{(9)}$.

El cuerpo de los textos fue utilizado para el análisis, y el método analítico empleado fue el análisis del contenido ${ }^{(10)}$. Se realizó una segmentación del texto, eligiendo los siguiente bloques temáticos: la protagonista, el trabajo, la preparación, la muerte, vivencias, encarnizamiento terapéutico, emociones, afrontamiento, preocupaciones, excelencia.

\section{RESULTADOS}

Respecto a la protagonista (tabla 1) del artículo la inmensa mayoría de los estudiantes se identifican como ella, comparten sentimientos y la entienden. Sienten respeto y admiración por ella como "personaje" trabajo.

En relación al trabajo (tabla 1) desarrollado por la enfermera oncológica se puede apreciar que lo consideran difícil pero gratificante. Aparece varias veces en el texto el apoyo, actitud, cálida, etc, simplemente describen cómo debería ser la enfermera con los pacientes terminales según su punto de vista.

\begin{tabular}{|l|l|}
\hline \multicolumn{2}{|c|}{ TABLA 1: LA PROTAGONISTA Y EL TRABAJO EN LA ONCOLOGÍA } \\
\hline & $\begin{array}{l}\text { "Esta visión es muy parecida a la mía." } \\
\text { "Me parece una persona con muchos valores humanos y con } \\
\text { una fortaleza interna enorme, una persona que lo ha pasado } \\
\text { muy mal pero que poco a poco se ha ido haciendo cada vez } \\
\text { más fuerte y ha sabido diferenciar muy bien los diferentes } \\
\text { aspectos de estas enfermedades." }\end{array}$ \\
$\begin{array}{l}\text { LA } \\
\text { PROTAGONISTA } \\
\text {-COPINIÓN } \\
\text { SOBRE LA }\end{array}$ & "Estoy de acuerdo con ella" \\
\hline
\end{tabular}




\begin{tabular}{|c|c|}
\hline ENTREVISTADA) & $\begin{array}{l}\text { "Todo lo que cuenta es una visión muy personal, pero } \\
\text { realmente, con mi pequeña trayectoria profesional (en } \\
\text { prácticas) he visto muchas cosas que esta chica lo relata } \\
\text { muy bien" } \\
\text { "Ella cuenta que al principio le era difícil sobrellevar la } \\
\text { muerte de los pacientes y el drama familiar que lo rodea, y es } \\
\text { así como yo me siento." } \\
\text { "El relato de la Enfermera, me ha impactado". }\end{array}$ \\
\hline EL TRABAJO & $\begin{array}{l}\text { "Con mas desgaste mental y psíquico que puede tener esta } \\
\text { profesión tan ardua y amarga es gratificante e interesante". } \\
\text { "La figura de la enfermera, que es el apoyo y la ayuda para } \\
\text { enfrenta esta última etapa de la vida, en la que el paciente } \\
\text { espera obtener una actitud cálida, amable, apoyo emocional, } \\
\text { cuidado... } \\
\text { "Yo pienso que los enfermeros deberíamos ser como un } \\
\text { punto de apoyo de aquel paciente o familiar que realmente } \\
\text { nos esté pidiendo ayuda a gritos..." } \\
\text { "Reconozco lo difícil que es trabajar en una unidad de estas } \\
\text { características donde cada día tenemos que enfrentarnos a } \\
\text { la muerte, siendo capaces a la vez de ser lo más } \\
\text { profesionales posible y dando todo nuestro apoyo tanto } \\
\text { técnico como emocional sin esto afecte a nuestra vida } \\
\text { personal." }\end{array}$ \\
\hline
\end{tabular}

Se puede observar (tabla 2) cómo piden "por gritos" la formación adecuada para poder enfrentarse con eficacia a situaciones que se pueden vivir en torno de la muerte.

Analizando el bloque de la muerte (tabla 2) se percibe la muerte como algo negativo. Se puede observar también que diferencian la muerte de uno y del outro, cuál es más difícil afrontarlo e incluso hay estudiantes que quieren luchar para salvar lo insalvable. No ven a la muerte como algo natural. Otros sin embargo dicen "Debemos dejar ir... dejar ir en paz". Se podría concluir este apartado con que existe variabilidad sobre las emociones de este apartado.

En cuanto al bloque del encarnizamiento terapéutico (tabla 2) se puede observar una uniformidad sobre las opiniones. 


\begin{tabular}{|c|c|}
\hline \multicolumn{2}{|c|}{$\begin{array}{l}\text { TABLA 2: LA PREPARACIÓN , LA MUERTE Y EL ENCARNIZAMIENTO } \\
\text { TERAPEUTICO }\end{array}$} \\
\hline LA PREPARACIÓN & $\begin{array}{l}\text { "Nos deberían de formar para este tipo de situaciones" } \\
\text { "En esta profesión vemos tantas muertes que pienso que } \\
\text { tenemos que estar preparados sobre este aspecto:- ¿Quién } \\
\text { nos enseña a decir "tu familiar a muerto" o a la pregunta de } \\
\text { uno de ellos "¿Por qué mi padre?”O el propio paciente } \\
\text { “Me voy a morir? } \\
\text { “Deberíamos tener una formación pregrado y postgrado } \\
\text { tanto de la muerte, como del paciente terminal, del duelo y } \\
\text { de cómo tratar a los familiares." } \\
\text { "No se preparan a los profesionales lo suficiente para } \\
\text { afrontar a diario la perdida de pacientes" } \\
\text { "Un primer paso en la mejoría de la formación de los } \\
\text { profesionales de la salud en general y de enfermería en } \\
\text { particular, consistiría en conocer las propias actitudes y } \\
\text { emociones hacia la propia muerte y hacia la muerte de los } \\
\text { demás." } \\
\text { "Es importante la formación en bioética y la formación en el } \\
\text { manejo de emociones.” }\end{array}$ \\
\hline LA MUERTE & $\begin{array}{l}\text { "Creo que la muerte no es plato de buen gusto para nadie." } \\
\text { "Creo que es algo a lo que nadie de nuestra profesión se } \\
\text { acostumbra con el tiempo" } \\
\text { "La muerte está muy rechazada por la sociedad y toda la } \\
\text { gente mira para otro lado o prefiere no hablar del tema" } \\
\text { "Veamos la muerte como la veamos, lo que parece } \\
\text { indiscutible es la perdida de una persona, y eso es lo que nos } \\
\text { hace tener miedo a muchas personas." } \\
\text { La "muerte", es una palabra muy dura y que incluso suena } \\
\text { mal. " } \\
\text { "Creo que nadie se acostumbra a ver morir a personas que } \\
\text { has ayudado, durante meses e incluso durante años, a } \\
\text { superar la enfermedad, que las coges mucho cariño y } \\
\text { aprecio, conoces muchos de sus secretos y confidencias y } \\
\text { sabes toda la historia familiar que hay detrás de la } \\
\text { enfermedad." }\end{array}$ \\
\hline
\end{tabular}




\begin{tabular}{|c|c|}
\hline & $\begin{array}{l}\text { "Cada caso es diferente, ya que la muerte de una persona de } \\
85 \text { años que está agonizando es mucho menos dolorosa que } \\
\text { la muerte de un niño". } \\
\text { "Personalmente, me sigue costando entender que tenga que } \\
\text { sentirme bien (o al menos satisfecha o tranquila) cuando un } \\
\text { paciente muere. Si bien es cierto que nos empleamos a fondo } \\
\text { para ofrecer todos los cuidados y comodidades a nuestros } \\
\text { pacientes terminales en su proceso de enfermedad, aún sigue } \\
\text { siendo difícil la idea de que nada va a cambiar el desenlace." } \\
\text { "Debemos dejar ir...dejar ir en paz." } \\
\text { "La población prefiere una muerte rápida, indolora, cuando } \\
\text { uno duerme.... Ya que no se teme solo a la muerte sino que } \\
\text { también al proceso que conlleva a ella." } \\
\text { "La mayoría de las personas prefieren una muerte en el } \\
\text { hospital ". } \\
\text { "En mi opinión la muerte siempre ha sido y será una de las } \\
\text { cosas que el ser humano piensa en algún momento de su } \\
\text { vida de forma racional, ya sea de manera religiosa, filosófica } \\
\text { o científica; y este pensamiento va a causar generalmente } \\
\text { sentimientos de angustia, miedo, respeto, ansiedad...." }\end{array}$ \\
\hline $\begin{array}{l}\text { EL } \\
\text { ENCARNIZAMIENTO } \\
\text { TERAPEUTICO }\end{array}$ & $\begin{array}{l}\text { "Nuestra implicación personal en nuestro trabajo puede } \\
\text { derivar en el encarnizamiento terapéutico, aunque éste } \\
\text { puede estar condicionado por los propios deseos del paciente } \\
\text { o de sus familiares." } \\
\text { "La forma de tratar debería de ser igual pero no lo es. A } \\
\text { una persona joven se le hace todo lo posible hasta el último } \\
\text { minuto (analítica, vías, tratamientos invasivos y agresivos) } \\
\text { pero, ¿es lo correcto? Ya que todo el equipo sanitario sabe } \\
\text { que ese paciente se va a morir, queramos o no, y no se le } \\
\text { deja descansar en paz, con su familia, sin dolor y con } \\
\text { tranquilidad. Creo que un encarnizamiento terapéutico no } \\
\text { es necesario en un caso de estas características ya que el } \\
\text { paciente está sufriendo más." } \\
\text { "Hay que evitar ensañamiento terapéutico y para orientar a } \\
\text { los profesionales sanitarios en el proceso de comunicación y } \\
\text { afrontamiento de los distintos casos y procesos." }\end{array}$ \\
\hline
\end{tabular}

Si observamos las vivencias personales (tabla 3) hablan de dolor, cariño, tristeza, de pasarlo mal, de poca ética, de parte de algunas enfermeras. Muchas veces la frecuencia con la que la enfermera se encuentra con la muerte puede llegar a endurecer y trivalizar con la muerte. 
A la hora de estudiar las emociones se encuentra la (tabla 3) con palabras como impacto emocional, dolor, impotencia, tristeza. La muerte no se puede concebir como un fracaso de la medicina, sino una etapa inevitable del ciclo vital.

Para las enfermeras el encuentro diario con la muerte y con el sufrimiento ajeno es como lidiar con una inmensa carga de emociones y desenvolverse en situaciones sin implicarse emocionalmente pero sin parecer frívolos. Es bastante difícil distinguir una actitud atenta y sensible pero no afectada de un cierta distanciamiento y frialdad que suele llevar a la rutina.

\begin{tabular}{|c|c|}
\hline \multicolumn{2}{|c|}{ TABLA 3: VIVENCIAS, EMOCIONES Y AFRONTAMIENTO } \\
\hline VIVENCIAS & $\begin{array}{l}\text { "En los hospitales en los que he estado de prácticas en } \\
\text { general es que las enfermeras (con las que he estado aunque } \\
\text { no todas) al morir un paciente en la planta no les importa, } \\
\text { hacen comentarios muy inapropiados sobre el paciente que } \\
\text { acaba de fallecer o incluso a sus familiares". } \\
\text { "He estado con enfermeras que verdaderamente se } \\
\text { preocupaban de ese paciente moribundo y cuando llegaba } \\
\text { su hora lo pasaban realmente mal al enterarse de la noticia } \\
\text { porque de verdad les dolió su muerte." } \\
\text { "Conocí pacientes que estaban allí cuando llegué y murieron } \\
\text { casi al final de mi rotación y, puedo asegurar que, en dos } \\
\text { meses, el roce y el cariño hacen mella dentro de una } \\
\text { enfermera. Dolía ver cómo iban apagándose y cómo la familia } \\
\text { lo sufría sin poder hacer nada pero, como buena profesional } \\
\text { que intentaba ser, insistía en los cuidados en la medida de lo } \\
\text { posible." } \\
\text { “Como vivencia personal, he de decir que he pasado por } \\
\text { situaciones bastante dolorosas en el tiempo que llevo } \\
\text { estudiando mi carrera. En segundo curso realicé prácticas } \\
\text { en Hematología, en un Hospital Oncológico. He de decir } \\
\text { que, aunque pasé días bastante malos, fue una experiencia } \\
\text { preciosa enormemente enriquecedora! }\end{array}$ \\
\hline EMOCIONES & $\begin{array}{l}\text { El impacto emocional que causa la muerte es mucho más } \\
\text { fuerte ahora que antes" } \\
\text { "Creo que la enfermera que le duele la pérdida de un ser } \\
\text { humano que además le ha estado cuidando en esa etapa (un } \\
\text { mes, dos meses) es la verdadera enfermera" } \\
\text { "Se crea un vínculo de unión de bondad, de voluntad, de }\end{array}$ \\
\hline
\end{tabular}




\begin{tabular}{|c|c|}
\hline & $\begin{array}{l}\text { empatía en definitiva de la labor propia de Enfermeria de } \\
\text { cuidar al otro. Cuando tienes relación con alguien al irse esa } \\
\text { persona que quieres no puedes evitar sentir impotencia, } \\
\text { desanimo, tristeza, melancolía" } \\
\text { "En mi opinión, para la enfermería puede resultar frustrante } \\
\text { la idea de perder a un paciente después de haber } \\
\text { proporcionado todos los cuidados que estaban a nuestro } \\
\text { alcance. Y no solo eso; las enfermeras sufrimos la pérdida por } \\
\text { la implicación que ya nos ha unido con nuestro paciente en su } \\
\text { proceso de enfermedad. " } \\
\text { "Los profesionales sanitarios, presos de su propio entorno } \\
\text { cultural, tienen también miedo a la muerte, a veces más que } \\
\text { los propios enfermos." } \\
\text { "Nuestros sentimientos, muchas ocasiones pueden beneficiar } \\
\text { o perjudicar nuestro trato con el enfermo. Nuestro grado de } \\
\text { afectividad influye claramente en nuestra profesionalidad } \\
\text { estando condicionado por cuestiones tan básicas como la } \\
\text { educación, la cultura, costumbres, religión..." }\end{array}$ \\
\hline AFRONTAMIENTO & $\begin{array}{l}\text { "También cuando habla de separar estoy de acuerdo con } \\
\text { ella, pero no sé porque tengo el temor de que el día de } \\
\text { mañana no sea yo capaz de hacerlo, imagino que si que } \\
\text { podré pero siempre tengo la duda. " } \\
\text { "Cada persona vivimos la muerte diferente, lo afrontamos } \\
\text { de diferente manera, unos tardan poco en asumirlo y otros } \\
\text { pueden tardar hasta años, el proceso del duelo depende de } \\
\text { cada persona." } \\
\text { "Realmente es difícil superar que una persona muera pero } \\
\text { más difícil es que muera con dolor " } \\
\text { "Todas estas situaciones son muy difíciles de llevar y es muy } \\
\text { difícil no llevarse los problemas del trabajo a casa pero se } \\
\text { debe intentar, ya que aunque tratemos a los pacientes como } \\
\text { mejor sabemos y con el mayor cariño posible no debemos } \\
\text { confundir los problemas familiares con los problemas de } \\
\text { trabajo ya que si todo lo del hospital nos influye en nuestra } \\
\text { vida diaria tenemos un problema." } \\
\text { "Mi madre, fue un gran apoyo. Ella, que tiene un largo rodaje } \\
\text { en el cuidado del enfermo oncológico, me dio muchos } \\
\text { consejos que me ayudaron (o eso creo) a ver las cosas desde } \\
\text { un ángulo diferente. Me hizo ver que la muerte estaba ahí de } \\
\text { todas formas con o sin las enfermeras.” }\end{array}$ \\
\hline
\end{tabular}


Analizando el bloque de "Afrontamiento" (tabla 3) se puede añadir que la muerte pone limitaciones al ser humano y al profesional. Muchas veces aparecen problemas de afrontamiento debido al rechazo que provoca la idea de la muerte. Dependiendo de cómo haya afrontado sus propias pérdidas elaborará sus propias estrategias de afrontamiento. En las unidades de terminales suelen organizar seminarios, grupos de debate, etc.

En relación a las "Preocupaciones" (tabla 4) se enfatiza tanto los propios sentimientos como la preocupación de los tipos de comunicación que se deberían adoptar en cada caso.

\begin{tabular}{|c|c|}
\hline \multicolumn{2}{|c|}{ TABLA 4: PREOCUPACIONES Y LA BÚSQUEDA DE LA EXCELENCIA } \\
\hline PREOCUPACIONES & $\begin{array}{l}\text { "Nadie te advierte todo los sentimientos que te van a ir } \\
\text { surgiendo y todo esos momentos que vas a vivir y a la vez te } \\
\text { van hacer tan fuerte, por suerte o por desgracia los vas } \\
\text { conociendo tu poco a poco y te vas mentalizando de todo } \\
\text { ellos, después de cómo en mi caso, llorar muchas veces." } \\
\text { "Creo que personalmente el que más me costaría sería la } \\
\text { comunicación, tanto con el paciente como con los familiares, } \\
\text { ya que hay momentos muy duros en los que tu vas a estar } \\
\text { con ellos, y en esos momentos, qué le dices, yo soy de las que } \\
\text { le da mas valor a una caricia y a un contacto que ha una } \\
\text { frase, ya que en esos momentos prácticamente todo está } \\
\text { dicho." }\end{array}$ \\
\hline $\begin{array}{l}\text { LA BÚSQUEDA DE } \\
\text { LA EXCELENCIA }\end{array}$ & $\begin{array}{l}\text { "Nosotras como enfermeras, no solo ponemos medicación y } \\
\text { hacemos técnicas si no que tenemos que tender una mano al } \\
\text { paciente cuando lo necesite, hablar con él, escucharle, } \\
\text { sonreírle, hacerle masaje en la espalda, hidratarle...todo } \\
\text { cuanto esté en nuestra mano para que se sienta mejor." } \\
\text { "Simplemente debemos pensar que hemos dado una calidad } \\
\text { de cuidados óptima a la persona dentro de sus condiciones. Y } \\
\text { eso es precisamente lo que debe darnos tranquilidad: el saber } \\
\text { que hemos realizado bien nuestro trabajo." } \\
\text { "Hay que prestar cuidados con el fin de que el paciente } \\
\text { sienta la seguridad de que todo el esfuerzo que hacemos por } \\
\text { él es por su bienestar." }\end{array}$ \\
\hline
\end{tabular}

En el bloque "Búsqueda de la excelencia" (tabla 4) se percibe el interés sobre la humanización de los cuidados durante la atención de la muerte, sin afectarnos emocionalmente para poder prestar cuidados sin que estén afectadas nuestras actuaciones, y a la vez ser sensibles/perceptivos a las demandas y necesidades de los pacientes y de sus familiares para lograr que los cuidados les reporten el máximo 
beneficio.

\section{DISCUSIÓN}

Para la realización de este trabajo se centró en el mundo de la persona entrevistada versus el mundo del estudiante de enfermería que en tercero de la diplomatura sabe que tiene que enfrentarse a situaciones parecidas a lãs de la protagonista del artículo.

El análisis de los resultados en relación a la actitud hacia el trabajo con enfermos terminales y hacia la muerte se pone de manifiesto em los siguientes datos: escasa preparación, afrontamiento ineficaz, miedos, valores y creencias. Predomina la inquietud relacionada con el encarnizamiento terapéutico y con la actitud obstinada del equipo de salud para salvar lo insalvable. Los elementos anteriormente mencionados actúan sinérgicamente y modulan el proceso de la atención al enfermo terminal.

Los resultados ponen de manifiesto un grado muy elevado de implicación emocional de la enfermera en el cuidado del paciente moribundo. Se considera como pilar fundamental una preparación específica para adaptarse mejor al trabajo con los paciente terminales. Destaca el interés y la preocupación por la excelencia.

\section{CONCLUSIONES}

Actitud y miedo a la muerte en la enfermería puede afectar la calidad de los cuidados en los enfermos terminales y moribundos. Es muy importante y necesario llevar a cabo investigaciones para determinar las estrategias que hay que adoptar en la educación de los cuidados paliativos para reducir así la ansiedad y el miedo que puede producir la muerte en los estudiantes de enfermería. Existen numerosos estudios cuantitativos sobre el miedo, el estrés en los estudiantes de enfermería referente con la muerte donde rellenan diferentes escalas y se les aportan a las respuestas números. Se interpretan los datos obtenidos en \%, medias, medianas, con o sin identificación estadística. Pero qué pasa con las variables que no recoge la encuesta pero están allí de manera invisible. Es muy importante el análisis de los problemas de "en torno de la muerte" en los estudiantes de enfermería. La investigación cualitativa en este campo podría ser uno de las herramientas útiles para detectar inquietudes y preocupaciones.

Un programa para "Adoptar y fomentar la estrategias de afrontamiento eficaces" y formación en antropología de la muerte serian muy útiles en la preparación de los futuros enfermeros. En el proceso del cuidado del paciente moribundo, la enfermera también puede desarrollar trastornos que deberían detectarse para ayudar a disminuir la aparición del síndrome Bournout y fomentar la aceptación a "convivir con la muerte".

\section{REFERENCIAS BIBLIOGRÁFICAS}

1. Haskovcová H; Thanatologie 2000 ed.1 Praga p. 191

2. Chabanne, J-C. y Bucheton, D. (2002). Parler et écrire pour penser, aprendre et se construire. L'ecrit et l'oral réflexifs. Paris: Presses Universitaries de France.

3. Hegedus, K., Zana, A., Szalo, G.; Effect of end of life education on medical students'and healt care workers ' death attitude. Palliative Medicine, 2008, vol. 22, no 3, p. 264-269. 
4. Naderi, F., Esmaili, E.: Collet-Lester Fear of Death Scale Validation and Gender Based Comparison of Death Anxiety, Suicide Ideation and Life Satisfaction in University Students.Journal of Applied Scientific, 2009, vol. 9, no 18, s. 3308-3316.

5. Mares, J.; Strach z bolesti; teorie a empirické výzkumy. Bolest,2002 vol 2 № 1 pag, 17-27

6. Neimeyer, R.A.; Death anxiety handbook; Research, Instrumentation and Aplicacion; London Taylor\& Francis 1994 pag, 284

7. Mooney, D.C.: Construct validity of the Revied Collett- lester Fear of Death and Dying scale Australia Griffith University 1999, pag. 85

8. Gil P.R.; Ávila R, I.M.; Molero P. M.J. Una visión enfermera del proceso de la muerte Archivos de la memoria. Disponible en http://www.indexf.com/memoria/6/r40906.php

9. Mendoza B.R La escritura reflexiva como práctica cotidiana de los pre-adolescentes y los adolescentes españoles: situación actual y características asociadas.

Disponible en http://www.revistaeducacion.mec.es/re335/re335_29.pdf

10. Vávrová, S., Polepilová, R.: VýzKumné setrení o vzdelávání studentu pomáhajících profesí v oblasti thanatologie. Sociální práce, 2010, vol. 10, no 2, p. 71 80.

11. S.Taylor, R. Bogdan: Introducción a los métodos cualitativos de investigación 1998 Barcelona. Paidós. 